\title{
RESTRICTED LIE ALGEBRAS WITH SEMILINEAR $p$-MAPPINGS
}

\author{
ROLF FARNSTEINER
}

\begin{abstract}
Restricted Lie algebras $(L,[p])$ having the property that some power of the $p$-mapping $[p]$ is semilinear are investigated.
\end{abstract}

1. Introduction. Since restricted Lie algebras were first introduced by N. Jacobson there have been a number of publications on the numerous correlations between properties of the $p$-mapping and the structure of the underlying Lie algebra. It is the main objective of this paper to determine those restricted Lie algebras $(L,[p])$ where some power of the $p$-mapping is semilinear with respect to the corresponding power of the Frobenius-homomorphism of the base field. It is well known that abelian restricted Lie algebras belong to this class. Our major result illustrates that, in the finite-dimensional case, nilpotency is a necessary condition for $[p]^{n}$ to be semilinear for some $n \in \mathbf{N}$. However, the converse does not have general validity. Using our result in combination with a commutativity theorem due to I. N. Herstein we demonstrate that every simple associative $F$-algebra having a $p$-semilinear $p$-map is a field.

Throughout this paper, $F$ is assumed to be a field of positive characteristic $p . T$ denotes an indeterminate over $F$.

Given a vector space $V$ over $F$ and a field homomorphism $\sigma: F \rightarrow F$, a mapping $f$ : $V \rightarrow V$ is called semilinear with respect to $\sigma$ if

$$
f(\alpha x+y)=\sigma(\alpha) f(x)+f(y) \quad \forall \alpha \in F, \forall x, y \in V .
$$

Let $L$ be a Lie algebra over $F$. We consider the Lie algebra $L \otimes_{F} F[T]$ and define functions $\alpha_{i}^{(l)}$ by $\operatorname{ad}_{x \otimes T+y \otimes 1}^{\prime}(x \otimes 1)=\sum_{i=0}^{l-1} \alpha_{i}^{(l)}(x, y) \otimes T^{i}$ for $l \in N, x, y \in L$.

REMARK 1 . The following identities can be readily verified:

$$
\alpha_{i}^{(l+1)}(x, y)= \begin{cases}\operatorname{ad}_{x}\left(\alpha_{i-1}^{(l)}(x, y)\right)+\operatorname{ad}_{y}\left(\alpha_{i}^{(l)}(x, y)\right), & 1 \leqslant i \leqslant l-1, \\ \operatorname{ad}_{x}\left(\alpha_{l-1}^{(l)}(x, y)\right), & i=l, \\ \operatorname{ad}_{y}\left(\alpha_{0}^{(l)}(x, y)\right), & i=0 .\end{cases}
$$

Using induction on $l$ one can check the following facts:

$$
\alpha_{l-1}^{(l)}(x, y)=-\operatorname{ad}_{x}^{l}(y)
$$

Received by the editors May 31, 1983.

1980 Mathematics Subject Classification. Primary 17B50.

${ }^{1}$ Research supported by the Minna-James-Heineman Foundation. 
If $A$ is a commutative $F$-algebra we consider the Lie algebra $L \otimes_{F} A$ and obtain $\alpha_{i}^{(l)}(x \otimes a, y \otimes b)=\alpha_{i}^{(l)}(x, y) \otimes$ $a^{i+1} b^{l-i}$.

Let $L$ be a Lie algebra over $F$. A mapping [ $p$ ]: $L \rightarrow L$ is called a $p$-mapping if

(1) $\operatorname{ad}_{x}^{[p]}=\operatorname{ad}_{x}^{p} \forall x \in L$

(2) $(\alpha x)^{[p]}=\alpha^{p} x^{[p]} \forall \alpha \in F, \forall x \in L$,

(3) $(x+y)^{[p]}=x^{[p]}+y^{[p]}+\sum_{i=1}^{p-1} s_{i}(x, y)$ where the functions $s_{i}$ are given by

$$
\operatorname{ad}_{x \otimes T+y \otimes 1}^{p-1}(x \otimes 1)=\sum_{i=1}^{p-1} i s_{i}(x, y) \otimes T^{i-1} .
$$

Using methods of $[3, \mathrm{p}$. 187] it can be inductively verified that given an associative algebra $A$ the identity $(x+y)^{p^{n}}=x^{p^{n}}+y^{p^{n}}+\sum_{i=1}^{p^{n}-1} s_{i}^{(n)}(x, y)$ holds, where $\operatorname{ad}_{x \otimes T+y \otimes 1}^{p^{n}-1}(x \otimes 1)=\sum_{i=1}^{p^{n}-1} i s_{i}^{(n)}(x, y) \otimes T^{i-1}$. Note that in contrast with the case $n=1$ the $s_{i}$ are not uniquely determined in general. Let $(L,[p])$ be a restricted Lie algebra with restricted universal enveloping algebra $u(L) \supset L$. According to the above we obtain

$$
(x+y)^{[p]^{n}}=x^{[p]^{n}}+y^{[p]^{n}}+\sum_{i=1}^{p^{n}-1} s_{i}^{(n)}(x, y) \quad \forall x, y \in L .
$$

The subsequent well-known result will be needed in the sequel.

LEMMA 1.1. Let $(L,[p])$ be a restricted Lie algebra over $F$ and suppose that $A$ is a commutative F-algebra. Then there is one and only one p-map on $L \otimes_{F} A$ such that $(x \otimes a)^{[p]}=x^{[p]} \otimes a^{p} \forall x \in L \forall a \in A$.

We conclude this section with the following

RemarK 2. Let $A$ be an associative $F$-algebra. For $l \in \mathbf{N}$ we define functions $\lambda_{i}^{(l)}$ by $(x \otimes T+y \otimes 1)^{l}=x^{l} \otimes T^{l}+y^{l} \otimes 1+\sum_{i=1}^{l-1} \lambda_{i}^{(l)}(x, y) \otimes T^{i}, l \geqslant 2, x, y \in A$. A routine computation verifies the subsequent formulas:

$$
\lambda_{i}^{(l+1)}(x, y)= \begin{cases}x \lambda_{i-1}^{(l)}(x, y)+y \lambda_{i}^{(l)}(x, y), & 2 \leqslant i \leqslant l-1, \\ x y^{l}+y \lambda_{1}^{(l)}(x, y) & i=1, \\ y x^{l}+x \lambda_{l-1}^{(l)}(x, y), & i=1 .\end{cases}
$$

Given a commutative $F$-algebra $B$ induction on $l$ proves

$$
\lambda_{i}^{(l)}(x \otimes a, y \otimes b)=\lambda_{i}^{(l)}(x, y) \otimes a^{i} b^{l-i} \quad \text { in } A \otimes_{F} B .
$$

2. Conditions for semilinearity. If $(L,[p])$ is a restricted Lie algebra over $F[p]^{n}$ : $L \rightarrow L$ is called semilinear if it is semilinear with respect to the homomorphism $\alpha \mapsto \alpha^{p^{n}}$.

THEOREM 2.1. Let $(L,[p])$ be a restricted Lie algebra over $F$. Let $x$, $y$ be elements of $L$ such that $(x \otimes T+y \otimes 1)^{[p]^{n}}=x^{[p]^{n}} \otimes T^{p^{n}}+y^{[p]^{n}} \otimes 1$. Then $\operatorname{ad}_{x}^{p^{n}-1}(y)=0$.

Proof. Consider $u\left(L \otimes_{F} F[T]\right)$ the restricted universal enveloping algebra of $L \otimes_{F} F[T]$. Our assumption entails the validity of $(x \otimes T+y \otimes 1)^{p^{n}}=x^{p^{n}} \otimes T^{p^{n}}$ $+y^{p^{n}} \otimes 1$ in $u\left(L \otimes_{F} F[T]\right)$. From Remark 2 of the preceding section we obtain 
$0=\sum_{i=1}^{p^{n}-1} \lambda_{i}^{\left(p^{n}\right)}(x \otimes T, y \otimes 1)=\sum_{i=1}^{p^{n}-1} \lambda_{i}^{\left(p^{n}\right)}(x, y) \otimes T^{i}$. In the last equation we made use of the canonical identification $u\left(L \otimes_{F} F[T]\right) \simeq u(L) \otimes_{F} F[T]$. Hence $\lambda_{i}^{\left(p^{n}\right)}(x, y)=0,1 \leqslant i \leqslant p^{n}-1$. Note that by definition $\lambda_{i}^{\left(p^{n}\right)}(x, y)=s_{i}^{(n)}(x, y)$. In particular, $s_{p^{n}-1}^{(n)}(x, y)$ vanishes. Consequently,

$$
\begin{aligned}
\operatorname{ad}_{x}^{p^{n}-1}(y) & =-\alpha_{p^{n}-2}^{\left(p^{n}-1\right)}(x, y) \quad(\mathrm{cf} .(1.1)) \\
& =s_{p^{n}-1}^{(n)}(x, y)=0 .
\end{aligned}
$$

COROLlaRY 2.2. Let $(L,[p])$ be a restricted Lie algebra with center $3(L)$ over $F$ such that $[p]^{n}: L \otimes_{F} F[T] \rightarrow L \otimes_{F} F[T]$ is semilinear. Then $L^{[p]^{n}} \subset 3(L)$. If $L$ is finite dimensional, then $L$ is nilpotent.

Proof. By virtue of Theorem 2.1 we obtain $\operatorname{ad}_{x}^{[p]^{n}}=\operatorname{ad}_{x}^{p^{n}}=0$ for every $x \in L$. $L^{[p]^{n}}$ therefore lies centrally in $L$. In particular, $L$ is ad-nilpotent and Engel's Theorem yields the nilpotency of $L$ in case of $L$ being finite dimensional.

We are now able to give a necessary condition for the semilinearity of the mapping $[p]^{n}: L \rightarrow L$.

Corollary 2.3. Let $(L,[p])$ be a restricted Lie algebra over $F$ and let $n$ be a positive integer such that $\operatorname{card}(F) \geqslant p^{n}$. Then the following statements hold:

(1) If $x$ and $y$ are elements of $L$ such that

$$
(\alpha x+y)^{[p]^{n}}=\alpha^{p^{n}} x^{[p]^{n}}+y^{[p]^{n}} \quad \forall \alpha \in F,
$$

then $\operatorname{ad}_{x}^{p^{n}-1}(y)=0$.

(2) If $[p]^{n}: L \rightarrow L$ is semilinear, then $L^{[p]^{n}}$ is contained in $3(L)$ and $[p]^{t}$ is semilinear for $t \geqslant n$.

(3) If $[p]^{n}$ is semilinear and $L$ is finite dimensional then $L$ is nilpotent.

Proof. (1) For every $\alpha \in F$ there exists a homomorphism of restricted Lie algebras $f_{\alpha}: L \otimes_{F} F[T] \rightarrow L$ such that $f_{\alpha}(x \otimes \gamma)=\gamma(\alpha) x \quad \forall x \in L \quad \forall \gamma \in F[T]$. According to our general definition we have $(x \otimes T+y \otimes 1)^{[p]^{n}}-x^{[p]^{n}} \otimes T^{p^{n}}-$ $y^{[p]^{n}} \otimes 1=\sum_{i=1}^{p^{n}-1} s_{i}^{(n)}(x \otimes T, y \otimes 1)=\sum_{i=1}^{p^{n}-1} s_{i}^{(n)}(x, y) \otimes T^{i}$. Applying $f_{\alpha}$ to this equation and using our present assumption yields

$$
0=\sum_{i=1}^{p^{n}-1} \alpha^{i} s_{i}^{(n)}(x, y) \quad \forall \alpha \in F
$$

Let $\left(e_{j}\right)_{j \in J}$ be a basis of $L$ over $F$ and write $s_{i}^{(n)}(x, y)=\sum_{j \in J} \alpha_{i j} e_{j}, 1 \leqslant i \leqslant p^{n}-1$. Put $\gamma_{j}:=\sum_{i=1}^{p^{n}-1} \alpha_{i j} T^{i}$ then (*) gives $0=\sum_{j \in J} \gamma_{j}(\alpha) e_{j} \forall \alpha \in F$. Since $\operatorname{deg} \gamma_{j} \leqslant p^{n}-1$ we conclude $\gamma_{j}=0 \quad \forall j \in J$. This yields $0=\sum_{j \in J} e_{j} \otimes \gamma_{j}=\sum_{i=1}^{p^{n}-1} s_{i}^{(n)}(x, y) \otimes T^{i}$. Consequently, the condition of Theorem 2.1 is satisfied by the pair $(x, y)$ and we obtain the desired result.

(2) The first part of our assertion is an immediate consequence of (1). Let $t$ be an integer $\geqslant n$. We write $[p]^{t}=[p]^{t-n} \circ[p]^{n}$. By virtue of our assumption $[p]^{n}$ is semilinear. Since $[p]^{n}$ maps $L$ into its center and $\left.[p]^{t-n}\right|_{3(L)}$ is semilinear our assertion readily follows. The last claim is a direct consequence of Engel's Theorem.

The following example illustrates that the nilpotency of a Lie algebra does not necessarily entail the semilinearity of some power of $p$-mapping (cf. [4, p. 97]). 
Let $V=\bigoplus_{i=1}^{p} F v_{i}$ be a $p$-dimensional vector space. We view $V$ as an abelian Lie algebra. Consider the endomorphism $f \in \operatorname{End}_{F}(V)$ which is defined by means of $f\left(v_{i}\right)=v_{i+1}, 1 \leqslant i \leqslant p-1, f\left(v_{p}\right)=0$. Let $L:=V \oplus F f$ denote the semidirect product of $V$ and $F f$, i.e. $[\alpha f+v, \beta f+w]=\alpha f(w)-\beta f(v) \forall \alpha, \beta \in F, \forall v, w \in V$. Evidently, $L^{n}=f^{n-1}(V) \forall n \geqslant 2$. ( $L^{n}$ denotes the $n$th component of the lower central series of $L$.) In particular, $L^{p}=F v_{p}$ and $L^{p+1}=0$. The latter equation implies that $\operatorname{ad}_{x}^{p}=0 \forall x \in L$. Applying Theorem 11 of [3, p. 190], we conclude that there exists a $p$-mapping $[p]: L \rightarrow L$ such that $v_{i}^{[p]}=0,1 \leqslant i \leqslant p-1, v_{p}^{[p]}=v_{p}$, $f^{[p]}=0$. Observing the identity $\operatorname{ad}_{f \otimes T+v_{1} \otimes 1}^{n}(f \otimes 1)=-f^{n}\left(v_{1}\right) \otimes T^{n-1}$ we find $s_{i}\left(f, v_{1}\right)=0,1 \leqslant i \leqslant p-2$, and $s_{p-1}\left(f, v_{1}\right)=v_{p}$. Consequently, $\left(f+v_{1}\right)^{[p]}=f^{[p]}$ $+v_{1}^{[p]}+v_{p}=v_{p}$ and $\left(f+v_{1}\right)^{[p]^{n}}=v_{p} \neq f^{[p]^{n}}+v_{1}^{[p]^{n}} \forall n \in \mathbf{N}$. Hence $[p]^{n}: L \rightarrow L$ is not semilinear.

An element $x \in L$ is called $p$-nilpotent if $x^{[p]^{n}}=0$ for some $n \in \mathbf{N}$. Note that in the preceding example the set $N(L)$ of $p$-nilpotent elements is not a subspace of $L$. Let $x$ be an element of $L$ and let $(F x)_{p}$ denote the $p$-subalgebra of $L$ generated by $x$. Evidently, $(F x)_{p}=\sum_{i \geqslant 0} F x^{[p]^{i}}$. The element $x$ is called semisimple if $x \in\left(F x^{[p]}\right)_{p}$. If $L$ is nilpotent then every semisimple element $x \in L$ lies in the center $8(L)$.

THEOREM 2.4. Let $(L,[p])$ be a finite-dimensional restricted Lie algebra over an infinite, perfect field. Then the following statements are equivalent:

(1) There is an $n \in \mathbf{N}$ such that $[p]^{n}$ is semilinear.

(2) $L$ is nilpotent and $N(L)$ is a subspace of $L$.

Proof. (1) $\Rightarrow(2)$. According to Corollary 2.3, $L$ is nilpotent. It is clear that $N(L)$ is stable under the multiplication by scalars. Given $x, y \in N(L)$ we find $t \geqslant n$ such that $x^{[p]^{t}}=y^{[p]^{t}}=0$. Owing to Corollary $2.3[p]^{t}$ is semilinear, hence $x+y \in N(L)$.

$(2) \Rightarrow(1)$. Since $F$ is perfect, every element $x$ of $L$ decomposes into a sum $x=y+z$ with $y$ semisimple and $z$-nilpotent (cf. [4, Theorem V.7.2]).

According to our previous remark, there is a subspace $V \subset \exists(L)$ consisting of semisimple elements such that $L=V \oplus N(L)$. (Note that the sum of two commuting semisimple elements is semisimple.) Since $N(L)$ is finite dimensional there is an $n \in \mathbf{N}$ such that $N(L)^{[p]^{n}}=0$. Let $x_{1}=y_{1}+z_{1}, x_{2}=y_{2}+z_{2}$ be elements of $L$, $y_{i} \in V, z_{i} \in N(L), 1 \leqslant i \leqslant 2$. Due to the inclusion $V \subset 3(L)$ we obtain

$$
\left(x_{1}+x_{2}\right)^{[p]^{n}}=\left(y_{1}+y_{2}\right)^{[p]^{n}}+\left(z_{1}+z_{2}\right)^{[p]^{n}}=y_{1}^{[p]^{n}}+y_{2}^{[p]^{n}}=x_{1}^{[p]^{n}}+x_{2}^{[p]^{n}} \text {. }
$$

Consequently, $[p]^{n}$ is semilinear.

B. S. Chwe has shown in [1] that if $F$ is algebraically closed then every finitedimensional restricted Lie algebra $(L,[p])$, having the property that 0 is the only zero of the $p$-map, is abelian. This result does not hold over arbitrary base fields. However, we can infer commutativity from semilinearity.

A restricted Lie algebra $(L,[p])$ is called $p$-algebraic if $(F x)_{p}$ is finite dimensional for every $x \in L$.

COROLlaRY 2.5. Let $F$ be perfect such that $\operatorname{card}(F) \geqslant p^{n}$ and suppose that $(L,[p])$ is a p-algebraic restricted Lie algebra such that (1) $[p]^{n}: L \rightarrow L$ is semilinear (2) 0 is the only zero of $[p]$. Then $L$ is abelian. 
Proof. Let $x$ be an element of $L$. Then (2) holds for the finite-dimensional restricted Lie algebra $(F x)_{p}$. Since $F$ is perfect $[p]:(F x)_{p} \rightarrow(F x)_{p}$ is surjective, thus $x \in L^{[p]}$. Now Corollary 2.3 gives $L=L^{[p]^{n}} \subset \mathcal{3}(L)$.

3. The associative case. Every associative $F$-algebra $A$ carries the structure of a restricted Lie algebra, where $[a, b]=a b-b a$ and $a^{[p]}=a^{p}$. Corollary 2.3 allows us to apply a commutativity theorem due to I. N. Herstein.

TheOREM 3.1. Let $F$ be a field with $\operatorname{card}(F) \geqslant p^{n}$. Suppose that $A$ is an associative $F$-algebra such that (1) $a \mapsto a^{p^{n}}$ is semilinear; (2) $A$ does not possess nontrivial nil ideals. Then $A$ is commutative.

Proof. Owing to Corollary $2.3, a^{p^{n}}$ lies in the center of $A$ for every element $a$ of $A$. Now apply Theorem 3.2.2 of [2].

REMARK. In particular, any simple associative $F$-algebra with semilinear Frobenius homomorphism is a field.

ACKNOWLEDGEMENT. The author would like to take the opportunity to thank Professor G. P. Hochschild for his advice and guidance.

\section{REFERENCES}

1. B. S. Chwe, On the commutativity of restricted Lie-algebra, Proc. Amer. Math. Soc. 16 (1965), 547.

2. I. N. Herstein, Noncommutative rings, Carus Math. Monographs, No. 15, Math. Assoc. America, Washington, D. C., 1968.

3. N. Jacobson, Lie-algebras, Dover, New York, 1979.

4. G. B. Seligman, Modular Lie-algebras, Springer-Verlag, Berlin, Heidelberg and New York, 1967.

Department of Mathematics, Univerisity of California, Berkeley, California 94720

Current address: Department of Mathematics, University of California, Riverside, California 92521 